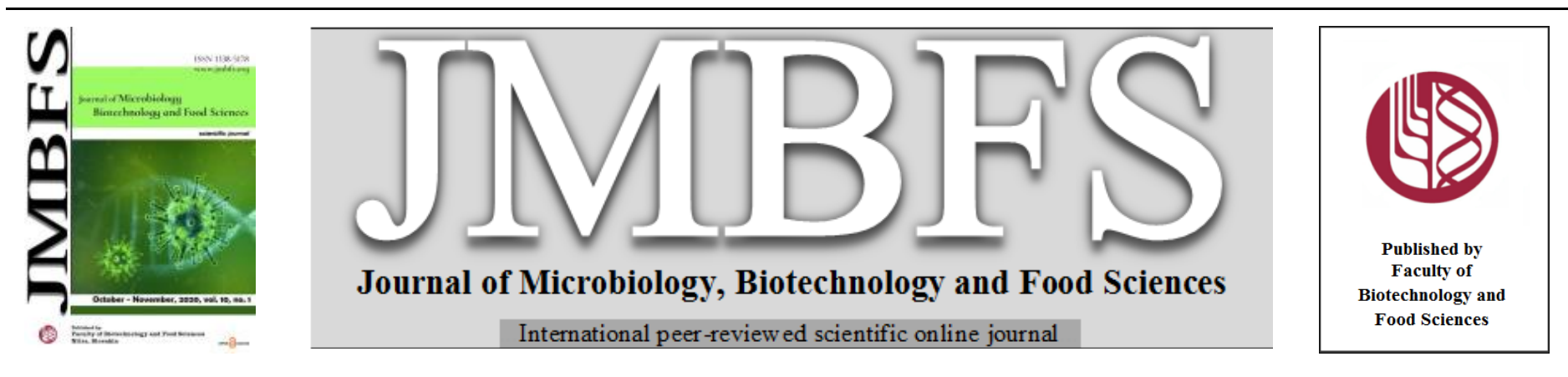

\title{
ISOLATION, SCREENING, AND CHARACTERIZATION OF BIOSURFACTANT-PRODUCING Bacillus SPp. FROM SOIL AND THEIR POTENTIAL BIOFILM INHIBITORY ACTIVITIES AGAINST Pseudomonas aeruginosa
}

\author{
John Paul Matthew D. Guzman*1,2, Jeun Marianne T. Alba ${ }^{2}$, Mark Louie S. Torres ${ }^{2}$ \\ Address(es): Mr. John Paul Matthew D. Guzman, \\ ${ }^{1}$ Environment and Biotechnology Division, Industrial Technology Development Institute, Department of Science and Technology, Bicutan, Taguig City, Philippines \\ 1631 \\ ${ }^{2}$ The Graduate School, University of Santo Tomas, España, Manila, Philippines 1008.
}

*Corresponding author: ipm.guzman@yahoo.com / ipmdguzman@itdi.dost.gov.ph

doi: 10.15414/jmbfs.2020.10.2.245-248

\section{ARTICLE INFO}

Received 15. 6. 2019

Revised 12. 6. 2020

Accepted 25. 6. 2020

Published 1. 10. 2020

$\underline{\text { Regular article }}$ OPEN $\partial_{\text {ACCESS }}$

\begin{abstract}
Biosurfactants are surface-active compounds usually produced by microbial cells, thus, their biodegradable nature and low toxicity. The capability to lessen surface and interfacial tensions characteristic of these compounds paved the way for their potential to inhibit biofilm formation. Biofilms are complex matrices of microbial cells formed on surfaces which provide microorganisms protection against substances found in the environment, including antimicrobials. In this study, Bacillus spp. isolated from soil samples were screened for their production of biosurfactants through Oil Drop Collapse and Parafilm M assays. Out of 12 isolates, four, GAT-01, GAT-04, GAT05, and GAT-07, tested positive, and were identified based on their phenotypic and genotypic characteristics as B. pseudomycoides, $B$. cereus, B. pseudomycoides, and B. mycoides, respectively. GAT-01 was able to yield the highest biofilm inhibition activity against Pseudomonas aeruginosa ATCC 27853 with $43.12 \%$, GAT-04 with 32.42\%, GAT-05 with 35.78\%, while GAT-07 showed the lowest activity with $26.91 \%$. No antibacterial activities against $P$. aeruginosa ATCC 27853 were observed. Quorum sensing inhibition assay using Chromobacterium violaceum ATCC 12472 also showed negative results for all the biosurfactants. These present the potential of biosurfactants from Bacillus spp. as bioactive substances against biofilm formation through physical interactions.
\end{abstract}

\section{INTRODUCTION}

Microorganisms are often described as the simplest form of organisms. However, further studies of microbial forms reveal that most are capable of complex differentiation and behavior (O'Toole, 2000). One of which is the capability of microorganisms to form biofilms. Biofilm refers to an interlinking network formed by multiple microorganisms that is attached on a surface (Donlan, 2001). It is considered a community of microorganisms that thrive on a surface that subsequently form a polymeric matrix (Costerton et al., 1999). This matrix often exhibits resistance to antibiotics; thus, biofilm formation is considered one of the many challenges in the field of medicine.

Currently, preventive strategies against biofilm formation include alteration of the surface coating or material of hospital instruments. However, recent studies suggest that molecules secreted within bacterial communities could interfere with formation of biofilm, thus limiting bacterial adhesion on surfaces (Valle et al., 2006). Several studies show promising results of biosurfactants against biofilm formation (Walencka et al., 2008). Biosurfactants are low molecular weight compounds produced by microorganisms capable of reducing surface and interfacial tensions. These structurally-diverse compounds include lipopeptides, glycolipids, and phospholipids among others. Their surface-active characteristic paved the way for their applications in bioremediation, food processing, oil recovery, and pharmaceuticals, to name a few. They are also highly regarded as more sustainable due to their capacity to endure a wider range of conditions, higher biodegradability, and low toxicity relative to their synthetic counterparts (Banat et al., 2000).

In this study, local isolates of Bacillus spp. from soil were screened for biosurfactant production and then tested for their capability to inhibit biofilm formation.

\section{MATERIAL AND METHODS}

\section{Soil smple collection}

Approximately 50 grams of top soil was collected along the perimeter of differen buildings in the Department of Science and Technology (DOST) Compound, Bicutan, Taguig City, Philippines namely: Environment and Biotechnology Division (EBD) Building, Standards and Testing Division (STD) Building, and National Metrology Laboratory (NML) Building. Collected soil samples were placed in properly-labeled sterile plastic bags and were immediately transported to the laboratory for analyses.

\section{Isolation of Bacillus spp.}

The soil samples were homogenized with sterile distilled water and two-fold serial dilution was done to obtain a $10^{-3}$ dilution. One hundred microliters of the soil suspension were inoculated onto Mannitol-Yolk-Polymyxin B (MYP) Agar and incubated at $35^{\circ} \mathrm{C}$ for $24 \mathrm{~h}$. Colonies visibly distinct from each other were selected and then isolated and purified on Nutrient Agar (NA) plates and were incubated at $35^{\circ} \mathrm{C}$ for $24 \mathrm{~h}$.

\section{Phenotypic identification of the isolates}

The colonial morphological characteristics of the isolates grown on NA plates were noted. Gram-staining was also done to characterize the cellular morphologies of the isolates. A number of biochemical tests were employed including lecithinase test, catalase test, oxidase test, indole test, methyl red test, Vogues-Proskauer test, citrate utilization and motility test. To further characterize and differentiate the isolates, their metabolic profiles were determined using a variety of carbohydrates including triple sugar iron agar test, mannitol, xylose, arabinose, and sorbitol. Isolates which showed characteristics typical of Bacillus species based on literature were selected. 


\section{Preparation of crude biosurfactants}

The crude biosurfactants were extracted according to the method of Hisham et al (2019) with few modifications. The isolates were grown statically in Tryptic Soy Broth (TSB) (Oxoid, UK) at $35^{\circ} \mathrm{C}$ for $24 \mathrm{~h}$. Afterwards, the cultures were centrifuged at $10,000 \mathrm{rpm}$ for $10 \mathrm{~min}$ and the supernatants were collected, and then filtered using $0.22-\mu \mathrm{m}$ syringe filter to obtain cell-free supernatants (CFS) The CFS of the isolates were used to screen for biosurfactants.

\section{Screening for biosurfactant production}

Oil dispersion assay

Twenty milliliters of distilled water was placed in a Petri dish, followed by the overlaying of $20 \mu \mathrm{L}$ of engine oil on the surface. Thereafter, $10 \mu \mathrm{L}$ of CFS was added onto the center of the pre-formed oil layer (Youssef et al., 2004). The oil displaced circles formed by CFS were visualized under visible light. TSB was used as the negative control in all the assays performed with CFS.

\section{Parafilm $M$ test}

Ten microliters of the CFS were dropped on the hydrophobic surface of a Parafilm M strip. The shape of the drop was observed after 1 min of incubation. The drop is positive for biosurfactants if it became flat, otherwise, if it remained dome-shaped, it indicates the absence of biosurfactants (Korayem et al., 2015). TSB served as the negative control.

\section{Molecular identification of biosurfactant-producing isolates}

The isolates which showed the ability to produce biosurfactants were selected for molecular identification. DNA extraction and PCR amplification were performed using Phire Plant Direct PCR Mastermix (Thermofisher Scientific, USA). The DNA sequences were amplified through PCR for 40 cycles using genera bacterial 16S rRNA primers 27F (5'-AGAGTTTGATCCTGGCTCAG-3') and 1492R (5'-GGTTACCTTGTTACGACTT-3'). The PCR conditions were as follows: Initial denaturation at $98^{\circ} \mathrm{C}$ for $5 \mathrm{~min}$; denaturation at $95^{\circ} \mathrm{C}$ for $5 \mathrm{~s}$; annealing at $58.6^{\circ} \mathrm{C}$ for $5 \mathrm{~s}$; extension at $72^{\circ} \mathrm{C}$ for $20 \mathrm{~s}$; and final extension at $72^{\circ} \mathrm{C}$ for $1 \mathrm{~min}$. The PCR products were then sent to Macrogen, Korea for sequencing analyses. BLAST search was done to determine the identity of the isolates based on gene sequence similarity. Phylogenetic tree was constructed through Maximum Likelihood algorithm with MEGA 7 software.

\section{Antibacterial activity}

\section{Determination of Minimum Inhibitory Concentration (MIC)}

Initially, a two-fold serial dilution of the crude biosurfactants $(100 \mu \mathrm{L})$ was prepared in a 96-well microtitre plate. Thereafter, $100 \mu \mathrm{L}$ of inoculum was added in each well. Similarly, TSB was also used as the negative control. Thereafter, the microtitre plates were incubated at $30^{\circ} \mathrm{C}$ for $24 \mathrm{~h}$. Growth was indicated by the presence of white pellets at the bottom of the wells. The MIC is defined as the lowest concentration that yielded inhibition of growth (Guzman et al., 2018).

\section{Determination of Minimum Bactericidal Concentration (MBC)}

To confirm the results of the MIC and to determine the MBC, the contents of the wells previously used to determine the MIC were streaked onto freshly-prepared TSA plates. The results of the MIC was confirmed when at least a single colony, an indication of growth, was observed. Meanwhile, the MBC was selected as the lowest concentration that showed absolutely no growth (Guzman et al., 2018).

\section{Agar well diffusion assay}

The agar well diffusion method was used to determine the antibacterial activity of the crude biosurfactants against $P$. aeruginosa ATCC 27853. Using a sterile cotton swab, standardized cell suspension was inoculated by spreading evenly over the surface of the Mueller-Hinton Agar (MHA). The plates were left to dry and a sterile cork borer $(5 \mathrm{~mm})$ was used to cut uniformly-sized wells in the agar. One hundred $(100 \mu \mathrm{L})$ of the different concentrations of the crude biosurfactants were added on each well. TSB also served as the negative control. The plates were then incubated at $30^{\circ} \mathrm{C}$ for $24 \mathrm{~h}$, and the zones of growth inhibition (ZOGI) were measured using a Vernier caliper (Guzman et al, 2018).

\section{Biofilm inhibition assay}

The crude biosurfactants were tested against $P$. aeruginosa ATCC 27853. First, a two-fold serial dilution of the crude biosurfactants $(100 \mu \mathrm{L})$ was prepared in a 96-well microtitre plate. TSB served as the negative control. Afterwards, $100 \mu \mathrm{L}$ of the inoculum was added in each well. The microtitre plates were incubated at $30^{\circ} \mathrm{C}$ for $24 \mathrm{~h}$. Thereafter, the contents of the wells were carefully discarded and washed with distilled water to remove planktonic cells. The biofilms adhering to the wells were then stained with $125 \mu \mathrm{L} 0.1 \%$ crystal violet for $15 \mathrm{~min}$. The wells were again washed to remove excess dye. The stained biofilms were dissolved using $125 \mu \mathrm{L} 30 \%$ acetic acid and were left for $15 \mathrm{~min}$ at room temperature to allow them to solubilize. Afterwards, the absorbance (OD $550 \mathrm{~nm}$ ) of each well was measured using a spectrophotometer to quantify the biofilms formed (O'Toole, 2011).

Percentage (\%) Biofilm Inhibition was computed using this formula (Karnjana et al., 2019):

$$
\% \text { Biofilm Inhibition }=\frac{(\text { AbsControl }- \text { AbsTreated })}{\text { AbsControl }}(100)
$$

\section{Quorum Sensing (QS) inhibition assay}

The crude biosurfactants were subjected to disk diffusion assay against Chromobacterium violaceum ATCC 12472. Standardized inocula were spread evenly and disks impregnated with the crude biosurfactants were placed on MHA. TSB served as the negative control. The plates were incubated at $30{ }^{\circ} \mathrm{C}$ for $24 \mathrm{~h}$. The presence of an opaque halo of which growth without pigmentation is observed signified the inhibition of QS (McClean et al., 1997).

\section{Statistical analysis}

Statistical analysis was performed using IBM SPSS Statistics 2.0 software. Oneway Analysis of Variance (ANOVA) was employed to determine the existence of significant differences among the ZOGIs and the absorbance values of the different concentrations of crude biosurfactants. Similarly, ANOVA was used to compare the treatments with the controls. To determine where the significant difference lies, Tukey's post hoc test was conducted.

\section{RESULTS AND DISCUSSION}

\section{Isolation of biosurfactant-producing bacteria from soil}

A total of 12 isolates were collected from the soil samples (Figure 1). The isolates were screened for biosurfactant production using Oil Dispersion Assay. One of the characteristics of a biosurfactant is its ability to alter surface tension (Banat et al., 2000). The results showed that the CFS of four isolates (GAT-01, GAT-04, GAT-05, and GAT-07) were able to disperse the oil layer on the surface of the water which indicate the presence of biosurfactants. This method is selected since it is able to detect even low concentrations of biosurfactants, which is suitable for the screening of biosurfactant production (Youssef et al., 2004).

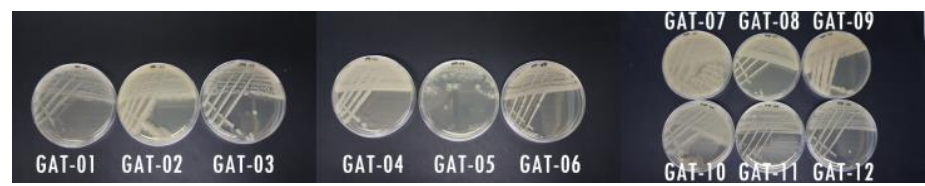

Figure 1 Presumptive Bacillus spp. from soil

To further verify the presence of biosurfactants, Parafilm M Test was employed The drops of CFS on Parafilm M strips were observed to become flat after incubation, thus, indicating the presence of biosurfactants (Figure 2). The results were due to the interaction between the biosurfactants present in the CFS and the hydrophobic surface of the Parafilm M strip (Korayem et al., 2015).

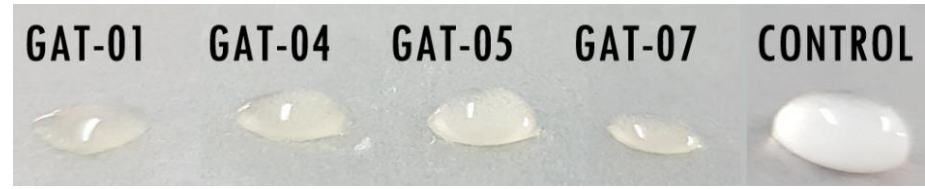

Figure 2 Parafilm M test.

\section{Phenotypic and genotypic identification of isolates}

The four biosurfactant-producing isolates (GAT-01, GAT-04, GAT-05, and GAT-07) were selected and preliminarily identified as Bacillus spp. based on their morphological and biochemical characteristics (Figure 3). Previous reports show that members of Bacillus spp. isolated from soil were capable of producing biosurfactants (Joshi et al., 2012; Zhou et al., 2015). 


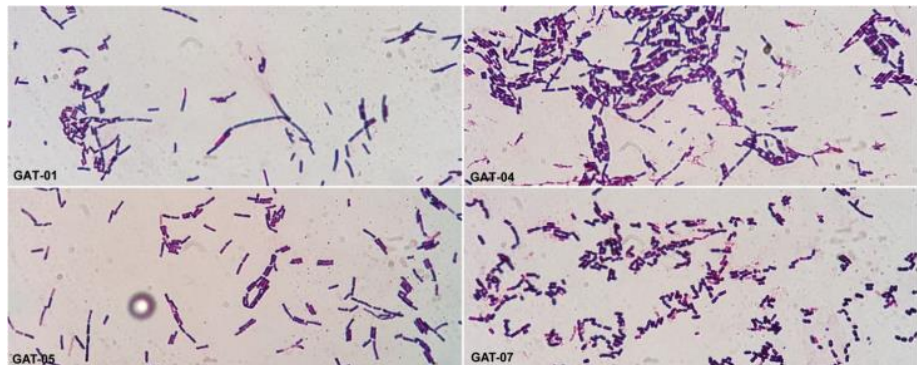

Figure 3 Gram-stain reaction of presumptive Bacillus spp. under the light microscope $(1000 x)$

Molecular identification through the amplification of 16S rRNA gene of the four selected biosurfactant-producing isolates was conducted (Figure 4). Based on sequence similarity, GAT-01 and GAT-05 were identified as B. pseudomycoides A strain of B. pseudomycoides isolated from oil-contaminated soil was previously observed to be a potent producer of biosurfactants. Further characterization of its biosurfactant showed that the compound is a cyclic lipopeptide (Li et al., 2016). Furthermore, isolate GAT-07 was identified as B. mycoides. A previous study on a $B$. mycoides strain isolated from an Iranian oil field showed its capability to produce a lipopeptide biosurfactant that was able to reduce surface tension by 34 $\mathrm{mN} / \mathrm{m}$ (Najafi et al., 2010). Lastly, GAT-04 was identified as B. cereus. An earlier report of a cadmium-tolerant $B$. cereus strain isolated from coffee grain showed its capability to produce biosurfactants composed mostly of proteins and lipids (Velasquez-Aradillas et al., 2011). A heavy metal-tolerant strain of $B$ cereus from a similar study was able to yield biosurfactants which were identified as a lipopeptide (Sriram et al., 2011). These results present the potential of exploiting locally-isolated strains of Bacillus spp. in the production of biosurfactants.

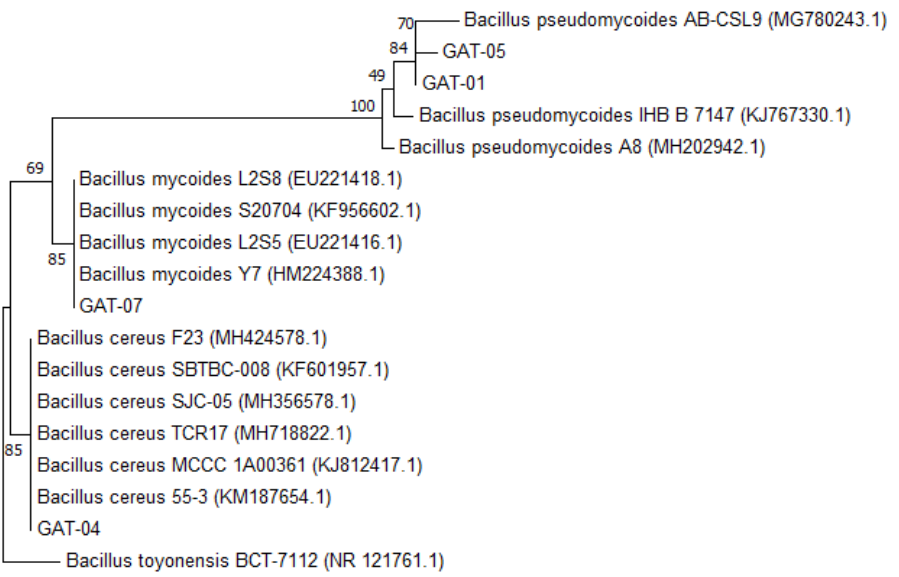

\subsection{0}

Figure 4 Phylogenetic tree of the biosurfactant-producing isolates.

\section{Antibacterial activity of Bacillus spp.}

The MICs of all the isolates were determined using two-fold serial dilution assay. The results showed that all the concentrations were able to yield growth as evident from the turbidity and white pellets at the bottom of the wells, hence, the MIC was not determined. To confirm the results of the MIC, the MBC of the isolates were also determined. After streaking the contents of the wells used in the determination of MIC onto freshly-prepared NA plates, growth was observed after incubation at all the concentrations. These results signified the possibility that the crude biosurfactants of all the isolates do not have any antibacterial activity against $P$. aeruginosa ATCC 27853 . However, previous studies showed that lipopeptide biosurfactant from Bacillus cereus NK1 have antimicrobial activities against select Gram-negative and Gram-positive organisms as well as against the yeast Saccharomyces cerevisiae (Sriram et al., 2011). Similarly, lipopeptide biosurfactant from Bacillus subtilis SPB1 showed antifungal activity against Rhizoctonia sp. (Mnif et al., 2015).

Hence, to further confirm the results, agar well diffusion assay was done. The different concentrations did not show any zones of inhibition. Therefore, the crude biosurfactants of all the isolates indeed do not have antibacterial activities. Nonetheless, crude biosurfactants were used in this study, wherein there might be negative pharmacodynamic interactions between the compounds present similar to those of the crude extracts in plants (Rasoanaivo et al., 2011), hence, the obtained results on the antibacterial assays.

\section{Biosurfactants from Bacillus spp. inhibit biofilm in P. aeruginosa}

The different concentrations of the four crude biosurfactants (GAT-01, GAT-04 GAT-05, GAT-07) were tested for their ability to inhibit biofilm formation on $P$. aeruginosa ATCC 27853 (Figure 5). The results showed that all four crude biosurfactants were able to significantly inhibit biofilm formation when compared with the control $(p<0.001)$. GAT-01 was able to inhibit biofilm formation even at the highest dilution $\left(10^{-9}\right)(p<0.001)$, and was able to yield the greatest activity $(43.12 \%)$ at the starting concentration $\left(10^{\circ}\right)$. Furthermore, both GAT-04 $(p<0.05)$ and GAT-05 $(p<0.001)$ were only able to inhibit until the 10 ${ }^{8}$ dilution. However, no significant difference $(p=0.296)$ was observed at the $10^{\circ}$ and $10^{-1}$ dilutions for GAT-04, signifying that at $10^{-1}$, the crude biosurfactants were able to yield the highest activity (32.42\%). Whereas, the starting concentration $10^{\circ}$ was able to yield the highest activity $(35.78 \%)$ for GAT-05 Similarly, GAT-07 was also able to inhibit biofilm formation until the highest dilution $10^{-9}(p<0.001)$ and was also able to yield the highest activity of $26.91 \%$ at the starting concentration $\left(10^{\circ}\right)$.

The results showed that GAT-01 yielded the highest activity among the crude biosurfactants used $(p<0.05)$. No significant differences, however, was observed between GAT-04 and GAT-05 $(p=0.439)$. Lastly, GAT-07 showed the lowest activity among the four crude biosurfactants $(p<0.05)$.

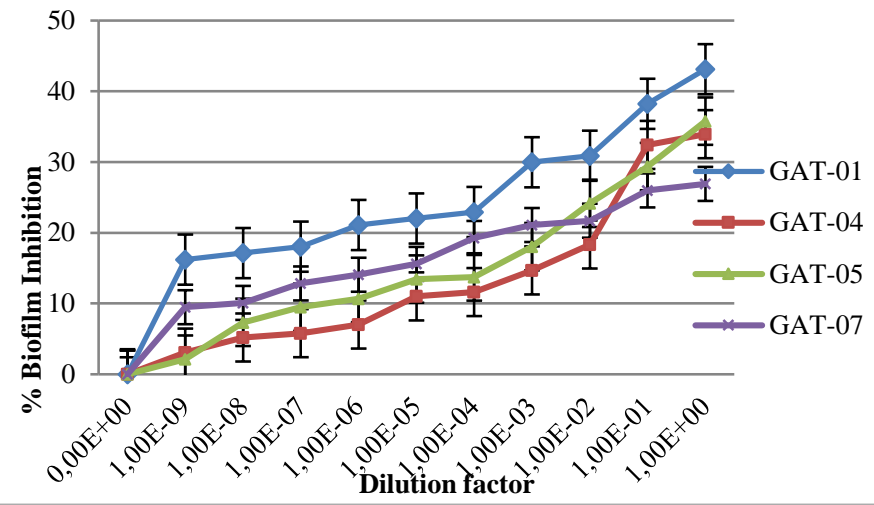

Figure 5 Biofilm inhibition activities of crude biosurfactants from Bacillus spp.

The results of the biofilm inhibition assays agree with previous studies that showed that biosurfactants from Bacillus sp. possess antibiofilm properties against a number of bacterial species (Freire et al., 2009; Rivardo et al., 2009). Moreover, a previous work states that purified lipopeptide biosurfactants from Bacillus sp. are capable of inhibiting biofilm formation in P. aeruginosa at around $40 \%$ to $50 \%$ (Sriram et al., 2011). Interestingly, one of the biosurfactants in this study, GAT-01, which yielded the highest activity among all tested biosurfactants, was able to exert an activity (43.12\%) comparable to that of previous studies. Therefore, the purification and isolation of the biosurfactants of this isolate may further enhance its biofilm-inhibitory activity.

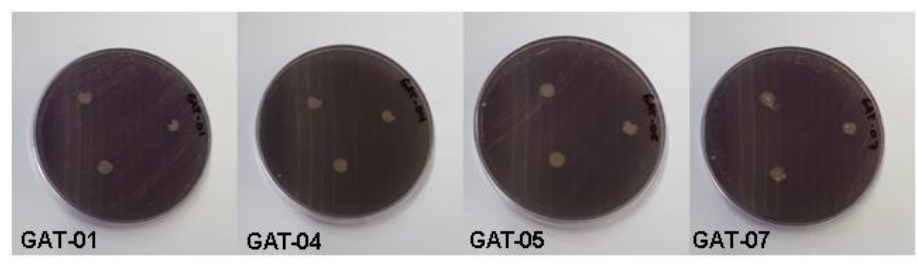

Figure 6 Quorum sensing inhibition assay of crude biosurfactants against $C$ violaceum ATCC 12472

To provide a putative mechanism as to how the crude biosurfactants were able to inhibit biofilm formation, quorum sensing inhibition (QSI) assay was done for all the biosurfactants in this study. Biofilm formation was proven to be dependent on quorum sensing, a cell-to-cell bacterial communication system exploiting the interactions between extracellular autoinducers and receptors found on the cells to initiate the transcription of a number of virulence factors including biofilm formation (Miller and Bassler, 2001). The results of the QSI assays using the biosensor strain $C$. violaceum ATCC 12472 revealed that the crude biosurfactants were not able to inhibit quorum sensing (Figure 6). Hence, the crude biosurfactants did not act on the different mechanisms involved in quorum sensing, but may have modulated the formation of biofilm through other means. Instead, these surface-active compounds may have lowered the hydrophobicity resulting in the exposure of negatively-charged groups of the surface (Quinn $e$ al., 2012). The biosurfactants may have also bound to the lipopolysaccharide of P. aeruginosa, thereby, increasing the net charge of the cells (Astuti et al., 2018) This resulted in the electrostatic repulsion between them and the negativelycharged surface, thus inhibiting the formation of biofilms or resulting in the formation of weak biofilms (Gomes and Nitschke, 2012; Kuiper et al., 2004). 


\section{CONCLUSION}

The growing interest in the novel means of combating bacterial infections, including inhibition of bacterial mechanisms, paved the way to look for new bioactive compounds. These include surface-active compounds produced by microorganisms called biosurfactants. In this study, four out of 12 isolated Bacillus spp. from different soil samples were able to produce biosurfactants. These crude biosurfactants also showed biofilm inhibitory activities against $P$. aeruginosa ATCC 27853, may be through the modulation of cell-surface hydrophobicity, with one of the biosurfactants yielding a relatively high activity comparable to that of previous studies. Hence, this shows the potential use of locally-isolated Bacillus spp. from soil in the production of biosurfactants and utilization in combating bacterial infections through the inhibition of biofilm formation. Isolation, purification, and further characterization of these compounds may enhance its effectiveness.

Acknowledgments: The researchers would like to express their gratitude to Prof. Paul Williams and Prof. Matthew Fletcher of the Centre for Biomolecular Sciences, University of Nottingham, United Kingdom for the biosensor strain; the Environment and Biotechnology Division of the Industrial Technology Development Institute, Department of Science and Technology for the assistance on the conduct of assays; Ms. Christine Eden Cortez of the Service Laboratory Group of the Food and Nutrition Research Institute for the help in the use of microplate reader; Dr. Thomas Edison dela Cruz of The Graduate School, University of Santo Tomas for his input; and Ms. Sheila Mantaring for proofreading the manuscript.

\section{REFERENCES}

Astuti, D., Purwasena, I., \& Putri, F. (2018). Potential of biosurfactant as an alternative biocide to control biofilm associated biocorrosion. J. Environ Sci Technol. 11:104-111. http://doi.org/10.3923/jest.2018.104.111

Banat, I., Makkar, R. \& Cameotra, S. (2000). Potential commercial applications of microbial surfactants. Appl. Microbiol Biotechnol, 53: 495-508 https://doi.org/10.1007/s002530051648

Costerton, J., Stewart, P. \& Greenberg, E. (1999). Bacterial Biofilms: A common cause of persistent infections. Science, 284: 1318-1322. http://doi.org/10.1126/science.284.5418.1318

Donlan, R. (2001). Biofilm Formation: A clinically relevant microbiological process. Healthcare Epidemiology: Clinical Infectious Diseases, 33: 13871392. https://doi.org/10.1086/322972

Freire, D., de Araujo, L., Kronemberger, F. \& Nitschke, M. (2009) Biosurfactants as emerging additives in food processing. Innovation in Food Engineering: New Techniques and Products. CRC Press: USA http://doi.org/10.1201/9781420086072-c23

Gomes, M., \& Nitschke, M. (2012). Evaluation of rhamnolipid and surfactin to reduce the adhesion and remove biofilms of individual and mixed cultures of food pathogenic bacteria. Food Control. 25(2): 441-447. https://doi.org/10.1016/j.foodcont.2011.11.025

Guzman, J., Cortes, A., Neri, K., Cortez, C., \& De las Alas, T. (2018) Antibacterial and Antibiofilm Activities of Sesbania grandiflora Against Foodborne Pathogen Vibrio cholerae. J App Pharm Sci, 8(03): 067-071. http://doi.org/10.7324/JAPS.2018.8310

Hisham, N, Ibrahim, M., Ramli, N., \& Abd-Aziz, S. (2019). Production of Biosurfactant Produced from Used Cooking Oil by Bacillus sp. HIP3 for Heavy Metals Removal. $\quad$ Molecules. 24(14): 1-16. https://doi.org/10.3390/molecules24142617

Joshi, S., Suthar, H., Yadav, A., Hingurao, K., \& Nerurkar, A. (2012). Occurrence of Biosurfactant Producing Bacillus spp. in Diverse Habitats. ISRN Biotechnol. 2013:1-6. http://doi.org/10.5402/2013/652340

Karnjana, K., Soowannayan, C. \& Wongprasert, K. (2019). Ethanolic extract of red seaweed Gracilaria fisheri and furanone eradicate Vibrio

harveyi and Vibrio parahaemolyticus biofilms and ameliorate the bacterial infection in shrimp. Fish Shellfish Immunol. 88:98- 101 http://doi.org/10.1016/j.fsi.2019.01.058

Korayem, A.S., Abdelhafez, A.A., Zaki, M.M. \& Saleh, E.A. (2015) Optimization of biosurfactant production by Streptomyces isolated from Egyptian arid soil using Plackett-Burman design. Ann. Agric. Sci. 60(2): 209-217. https://doi.org/10.1016/j.aoas.2015.09.001

Kuiper, I., Lagendijk, E., Pickford, R., Derrick, J., Lamers, G., Thomas-Oates, J. Lugtenberg, B. \& Bloemberg, G. (2004). Characterization of two Pseudomonas putida lipopeptide biosurfactants, putisolvin I and II, which inhibit biofilm formation and break down existing biofilms. Mol Microbiol, 51(1):97-113 https://doi.org/10.1046/j.1365-2958.2003.03751.x

Li, J., Deng, M., Wang, Y., \& Chen, W. (2016). Production and characteristics of biosurfactant produced by Bacillus pseudomycoides BS6 utilizing soybean oil $\begin{array}{lllll}\text { waste. Int } & \text { Biodeterior } & \text { Biodegradation. }\end{array}$ http://dx.doi.org/10.1016/j.ibiod.2016.05.002

McClean, K., Winson, M., Fish, L., Taylor, A., Chhabra, S., Camara, M., Daykin, M., Lamb, J., Swift, S., Bycroft, B., Stewart, G., \& Williams, P. (1997). Quorum sensing and Chromobacterium violaceum: Exploitation of violaceinproduction and inhibition for the detection of $\mathrm{N}$-acylhomoserine lactones. Microbiology. 143(12):3703-3711. https://doi.org/10.1099/00221287-143-12-3703

Miller, M. \& Bassler, B. (2001) Quorum sensing in bacteria. Annu Rev Microbiol. 55:165-199. https://doi.org/10.1146/annurev.micro.55.1.165

Mnif, I., Gran-Campistany, A., Coronel-Leon, J., Hammami, I., Triki, M. Manresa, A. \& Ghribi, D. (2015). Purification and identification of Bacillus subtilis SPB1 lipopeptide biosurfactant exhibiting antifungal activity against Rhizoctonia bataticola and Rhizoctonia solani. Environ Sci Pollut Res. 7:6690-6699. http://doi.org/10.1007/s11356-015-5826-3

Najafi, A., Rahimpour, M., Jahanmiri, A., Roostaazad, R., Arabian, D., \& Ghobadi, Z. (2010). Enhancing biosurfactant production from an indigenous strain of Bacillus mycoides by optimizing the growth conditions using a response surface methodology. Chem Eng J.

$163: 188-194$ http://doi.org/10.1016/j.cej.2010.06.044

O’Toole, G. (2011). Microtiter Dish Biofilm Formation Assay. JoVE. 47:1-2 http://doi.org/10.3791/2437

O’Toole, G., Kaplan, H. \& Kolter, R. (2000). Biofilm formation as microbial development. Annu Rev https://doi.org/10.1146/annurev.micro.54.1.49

Quinn, G., Maloy, A., McClean, S., Carney, B., \& Slater, J. (2012). Lipopeptide biosurfactants from Paenibacillus polymyxa inhibit single and mixed species biofilms. $\quad$ Biofouling, 28(10), 1151-1166. http://doi.org/10.1080/08927014.2012.738292

Rasoanaivo, P., Wright, C., Wilcox, M. \& Gilbert, B. (2011). Whole plant extract versus single compounds for the treatment of malaria: synergy and positive interactions. Malaria J. 10(1): 1-12. https://doi.org/10.1186/1475-2875-10-s1-s4

Rivardo, F., Turner, R., Allegrone, G., Ceri, H. \& Martinotti, M. (2009). Antiadhesion activity of two biosurfactants produced by Bacillus spp. prevents biofilm formation of human bacterial pathogens. Appl Microbiol Biotechnol 83:541-553. https://doi.org/10.1007/s00253-009-1987-7

Sriram, M., Kalishwaralal, K., Deepak, V., Gracerosepat, R., Sriskathi, K. \& Gurunathan, S. (2011). Biofilm inhibition and antimicrobial action of lipopeptide biosurfactant produced by heavy metal tolerant strain Bacillus cereus NK1. Colloids and $\mathrm{B}$ $85: 174-181$ https://doi.org/10.1016/j.colsurfb.2011.02.026

Tenover, F.C. (2009). Antibiotic susceptibility testing. In: Schaechter M, ed. Encyclopedia of Microbiology. 3rd ed. USA: Elsevier 67-77

Valle, J., Da Re, S., Henry, N., Fontaine, T., Balestrino, D., Latour-Lambert, P., \& Ghigo, JM. (2006). Broad-spectrum biofilm inhibition by a secreted bacterial polysaccharide. PNAS, 103(33): 12558-12563.

Velasquez-Aradillas, J., Toribio-Jimenez, J., Gonzalez-Chavez, M., Bautista, F., Cebrian, M., Esparza-Garcia, F., \& Rodriguez-Vazquez, R. (2011) Characterisation of a biosurfactant produced by a Bacillus cereus strain tolerant to cadmium and isolated from green coffee grain. World J Microbiol Biotechnol. 27:907-913. http://doi.org/1 0.1007/s11274-010-0533-1

Walencka, E., Rozalska, S., Sadowska, B. \& Rozalska, B. (2008). The influence of Lactobacillus acidophilus-derived surfactants on Staphylococcal adhesion and biofilm formation. Folia $\quad$ Microbiol, $\quad$ 53(1): 61-66 https://doi.org/10.1007/s12223-008-0009-y

Youssef, N., Duncan, E., Nagle, D., Savage, K., Knapp, R. \& McInerney, M (2004). Comparison of methods to detect biosurfactant production by diverse $\begin{array}{llll}\text { microorganisms. } & \mathrm{J} & \text { Microbiol } & \text { Met, }\end{array}$ https://doi.org/10.1016/j.mimet.2003.11.001

Zhou, H., Chen, J., Yang, Z., Qin, B., Li Y., \& Kong, X. (2015). Biosurfactant production and characterization of Bacillus sp. ZG0427 isolated from oilcontaminated soil. Ann Microbiol. 65(4): 2255-2264. http://doi.org/1 0.1007/s13213-015-1066-5 